\title{
PARTIAL ORDERINGS OF LIFE DISTRIBUTIONS BASED ON AGEING PROPERTIES
}

\author{
A. M. ABOUAMMOH, A. N. AHMED AND A. KHALIQUE
}

\begin{abstract}
A bstract. Some new generalized forms of partial orderings of life distributions are introduced. Some of these orderings are based on the virtual age such as decreasing virtual ordering, new better than used virtual ordering, decreaing average virtual ordering and new better than average virtual ordering. Similar types of orderings are defined in terms of the hazard rate. The interrelationships between these orderings are investigated and their relations with other known orderings such as the mean remaining life ordering and the global memory ordering are discussed. Some useful classes of life distributions are shown to arise when such orderings are considered with respect to the negative exponential distribution. Other related results are also discussed.
\end{abstract}

\section{Introduction.}

Selection of probability models for different phenomena such as the time to failure for a system or its units, repair time, waiting time in a queue and ageing ect., is performed by observing the underlying physical characteristics. Use of exponential distribution for modelling these phenomena is simple and widely used to represent the memoryless property. This ideal property has very limited real life applications. Model building researchers are interested in a more extended case that is nonnegative or simply involves positive ageing, see Bryson and Siddiqui (1969) and Barlow and Proschan (1981).

The main theme of this paper is to introduce some notions of positive ageing for evaluating the reliability of a device and to carryout some comparisons between the reliability of systems or their components in terms of these notions of ageing. One useful notion of ageing is the virtual age, that is, the difference between the mean remaining life at two distinct limits of time interval is nonnegative. Another practical notion is the global memory which is the rate of change of decreasing mean remaining age over the interval $(0, \infty)$. For some properties of virtual age and global memory, we refer to Muth (1980). The notion of virtual hazard rate is introduced since hazard rate is more likely to be observed in survival data, when the density functin of the underlying life distribution exists.

Received Oct. 18, 1988; revised March, 28, 1989. 
Various criteria of ordering life distributions are useful in different aspects such as comparing the tail of distributions in goodness of fit tests, see Bryson (1974), comparing the peakedness of life distrbutions, giving unified implications between life distrebutions when the ordering is made with repect to exponentiality and attributing physical meaning to various underlying models in stochastic processes.

The sequel of the paper is as follows: Some definitions and preliminary results are given in Section 2. Section 3 includes the partial orderings that are based on the concept of virtual age. In Section 4, some results concering the global memory ordering and the variability ordering are discussed. The virtual hazard rate based orderings are investigated in Section 5 and the last Section 6 includes some applications-oriented and miscellaneous results.

\section{Preliminaries.}

Let $T$ be a nonnegative random variable with distribution function $F(t)=P(T \leq t)$ and survival function $\bar{F}(t)=1-F(t)$. Let $F(t)$ be differentiable and $f(t)=-\left(\frac{d}{d t}\right) \overline{\bar{F}}(t)$ be the density function of $T$. The hazard rate $r_{F}(t)$ is defined by

$$
\begin{aligned}
r_{F}(t) & =\lim _{\delta t \rightarrow 0} \frac{1}{\delta t} P(T \leq t+\delta t \mid T>t) \\
& =\frac{f(t)}{\bar{F}(t)}
\end{aligned}
$$

The mean remaining life of the random variable $T$ is given by

$$
\begin{aligned}
\mu_{F}(t) & =E(T-t \mid T \geq t) \\
& =\int_{t}^{\infty} \frac{\bar{F}(u) d u}{\bar{F}(t)}
\end{aligned}
$$

The following two definitions are due to Muth (1980).

Definition 2.1. The virtual age of a life distrbution of age $t$, denoted by $v_{F}(t)$ is given by

$$
V_{F}(t)=\mu_{F}(0)-\mu_{F}(t)
$$

Defnition 2.2. The global memory of a life distribution $F$ denoted by $m_{g}(F)$ is given by

$$
m_{g}(F)=2-\frac{2}{\mu_{F}^{2}} \int_{0}^{\infty} \mu_{F}(u) \bar{F}(u) d u
$$

where $\mu_{F}=\mu_{F}(0)$ and $\mu_{F}($.$) is as given in relation (2.2).$

Note that a life distrbution $F$ is said to be of positive global memory (PGM) if $m_{g}(F) \geq 0$.

Next we present the mean remaining life ordering, see Kochar and Weins (1987). 
Definition 2.3. A life distribution $\mathbb{F}$ is said to be uniformly smaller mean remaining life than the life distrbution $G$, written $F<_{m r l} G$ if

$$
\mu_{F}(t) \leq \mu_{G}(t), \quad \text { for all } t \geq 0 .
$$

The following definition is due to Gupta and Keating (1986).

Definition 2.4. Let $T$ be a nonnegative random variable with finite mean $\mu$ and desity function $\mathrm{f}$. The length biased density of $T$ is given by

$$
f_{b}(t)=t \frac{f(t)}{\mu}, \quad t>0 .
$$

The length biased radom variable of $T$ is denoted $T_{b}$ and we have

$$
\bar{F}_{b}(t)=\frac{\bar{F}(t)}{\mu}(t+\mu(t)),
$$

and

$$
r_{b}(t)=\frac{t f(t)}{\bar{F}(t)(t+\mu(t))}
$$

where $\mu(t)$ is given by relation (2.2).

In the material that follows the terms increasing and decreasing stand for nondecreasing and non-increasing respectively.

Note that if $V_{F}(t)$ is increasing then $F$ is to have decreasing mean remaining life (DMRL) properiy. Whereas if $V_{F}(t) \geq 0$, then $F$ is said to have new better than used mean remaining life (NBUMRL) property, see Abouammoh (1988). If in Definition 2.3 $\bar{G}(t)=\exp \left(-\frac{t}{\mu_{F}}\right)$, then $F$ is NBUMRL as well.

Another interesting ordering which has many applications in stochastic processes, see Ross and Schechner (1984), is the following.

Definition 2.5. Let $F$ and $G$ be two life distributions. Then $F$ is said to be less variability ordered than $G$, denoted $F<{ }_{v} G$ if

$$
\int_{t}^{\infty} \bar{F}(u) d u \leq \int_{t}^{\infty} \bar{G}(u) d u \quad \text { for all } t \geq 0 .
$$

Note that if $\bar{G}(t)=\exp \left(\frac{-t}{\mu_{F}}\right), \quad t \geq 0$, in (2.9) then $F$ is said to have harmonic new better than used in expectation (HNBUE) property, see Rolski (1975).

Next we present the following definition.

Definition 2.6. Let $F$ and $G$ be two absolutely continuous life distributions with respective failure rates $r_{F}(t)$ and $r_{G}(t)$, then $F$ is said to have uniformly smaller hazard rate than $G$, denoted by $F<_{h} G$ if

$$
r_{F}(t) \leq r_{G}(t), \quad \text { for all } t \geq 0 .
$$


This ordering is also know as the $T P_{2}$ ordering, see Bagai and Kochar (1986) and is denoted by $F<_{T P_{2}} G$.

Definition 2.7. The life distribution $F$ is said to be normalized mean residual life oedered with respect to $G$, denoted by $F<_{n m r 1} G$, if

$$
\mu_{G} \mu_{F}(t) \leq \mu_{F} \mu_{G}(t), \quad \text { for all } t \geq 0 \text {. }
$$

\section{Virtual life orderings.}

Let $F$ and $G$ be two distribution functions of nonnegative random variables such that $F(0)=G(0)=0$. Now we introduce the following definition.

Definition 3.1. Let $V_{F}(t)$ and $V_{G}(t)$ be the virtual ages of distibution $F$ and $G$, respectively. Then we have the following:

(i) $F$ is less virtual decreasing (vd) ordered than $G$, denoted by $F<_{v d} G$ if the difference $V_{F}(t)-V_{G}(t)$ is decreasing in $t$ that is

$$
\left[V_{F}(t)-V_{G}(t)\right] \searrow \text { in } t, \quad t \geq 0
$$

(ii) $F$ is less new better than used virtual (nbuv) ordered than $G$, denoted by $F<_{n b u v} G$ if

$$
V_{F}(t) \leq V_{G}(t), \quad \text { for all } t \geq 0,
$$

(iii) $F$ is less averaged decreasing virtual (adv) ordered than $G$, denoted by $F<_{a d v} G$ if,

$$
t^{-1} \int_{0}^{t}\left[V_{F}(u)-V_{G}(u)\right] d u \searrow \text { in } t, \quad \text { for all } t \geq 0 \text {. }
$$

(iv) $F$ is less new better than averaged used virtual (nbav) ordered than $G$, denoted by $F<_{\text {nbav }} G$, if

$$
\int_{0}^{t} V_{F}(u) d u \leq \int_{0}^{t} V_{G}(u) d u, \quad \text { for all } t \geq 0 .
$$

It can be easily shown that the orderings in Definition 3.1 are partial orderings since they are reflexive, antisymmetric and transitive. Now we give the following characterization of some classes of life distrbutions in terms of virtual orderings.

Theorem 3.2. Let $G(t)=1-\exp (-\lambda t)$ be an exponential distribution and $F$ be any distrbution function with $F(0)=0$. Then

(i) $F>_{v d} G$ if and only if (iff) $F$ has the decreasing mean remaining life (DMRL) property. 
(ii) $F>_{\text {nbuv }} G$ iff $F$ has the new better than used mean remaining life (NBUMRL) property.

(iii) $F>_{a d v} G$ iff $F$ has the decreasing mean remaining life average (DMRLA) property.

(iv) $F>_{n b a v} G$ iff $F$ has the new better than averaged mean remaining life (N.BAMRL) property.

Proof. Parts (i), (ii) and (iv) are obvious whereas the proof of part(iii) is as follows. (iii) Since $t^{-1} \int_{0}^{t} V_{G}(u) d u=$ constant, we get

$$
\begin{gathered}
\\
t^{-1} \int_{0}^{t} V_{G}(u) d u \nearrow \text { in } t \\
\Leftrightarrow t^{-1} \int_{0}^{t} \mu_{F}(u) d u \searrow \text { in } t \\
\text { i.e., } \quad F \text { is DMRLA. }
\end{gathered}
$$

Nex we give the forrowing two results whose proof are straightforward.

Theorem 3.3. Let $\mu_{F}=\mu_{G}$ then $F>_{m r l} G$ iff $F<_{\text {nbuv }} G$.

Theorem 3.4. Let $F$ and $G$ have the same mean $\mu, F>_{n b u v} G$ and $G$ is NBUE, then $F$ is NBUE.

The second result shows that the NBUE property is preserved under the nbuv ordering.

Using the fact that the derivative of a decreasing function is nonpositive one can prove the following theorem.

Theorem 3.5.

(i) $F$ is $D M R L$ iff $\mu_{F}(t) r_{F}(t) \leq 1$.

(ii) $F<_{v d} G$ iff $\mu_{F}(t) r_{F}(t) \geq \mu_{G}(t) r_{G}(t)$.

(iii) $F<_{a d v} G$ iff $w(t) \leq t^{-1} \int_{0}^{t} w(u) d u$, where $w(t)=V_{F}(t)-V_{G}(t)$.

4. Global memory orderings.

First, we define the global memory orderings.

Denfinition 4.1. The life distribution $F$ is said to be less global memory ordered then $G$, denoted by $F<_{g} G$ if

$$
m_{g}(F) \leq m_{g}(G)
$$

New, we prove the following.

Theorem 4.2. Let $F$ and $G$ be two life distributions with equal means and $F<_{v} G$ then $F<_{g} G$. 
Proof. It is given that $\mu_{F}=\mu_{G}$ and $\int_{t}^{\infty} \bar{F}(u) d u \leq \int_{t}^{\infty} \bar{G}(u) d u$.

$$
\begin{aligned}
m_{g}(F) & =2-\frac{2}{\mu_{F}^{2}} \int_{0}^{\infty} \int_{t}^{\infty} \bar{F}(u) d u d t \\
& \leq 2-\frac{2}{\mu_{G}^{2}} \int_{0}^{\infty} \int_{t}^{\infty} \bar{G}(u) d u d t \\
& =2-\frac{2}{\mu_{G}^{2}} \int_{0}^{\infty} \mu_{G}(t) \bar{G}(t) d t \\
& =m_{g}(G) .
\end{aligned}
$$

Integrating (4.2) by parts with respect to $t$ gives that

$$
m_{g}(F)=2-\mu_{F}^{-2} E_{F} T^{2}
$$

Using Theorem 4.2 and relation (4.3), we obtain the following.

Corollary 1. Under the assumptions of Theorem 4.2,

$$
\operatorname{Var} F \geq \operatorname{Var} G
$$

Corollary 2. Let $C_{F}$ be the coefficient of variation of the distribution $F$, then

$$
C_{F}^{2}=1-m_{g}(F)
$$

Theorem 4.3. Let the life distrbution $F$ be NBUMRL then $m_{g}(F) \geq m_{g}(G)$ where $\bar{G}(t)=\exp \left(\frac{-t}{\mu}\right), \mu>0, t \geq 0$, i.e., $F$ is $P G M$ or $m_{g}(F) \geq 0$.

Proof. Let $F$ be NBUMRL distribution that is $\mu(t) \leq \mu$, which is equivalent to

$$
\mu_{F}(t) \bar{F}(t) \leq \mu \bar{F}(t), \quad \text { for all } t \geq 0
$$

The relation (4.6) implies

$$
\int_{0}^{\infty} \mu_{F}(t) \bar{F}(t) d t \leq \mu^{2}
$$

This means that $m_{g}(F) \geq 0$.

In the following theorem we show that the PGM class is wider than the HNBUE class.

Theorem 4.4. Let $F$ be a HNBUE life distribution then $F$ is PGM. 
Proof. $F$ is HNBUE, from Rolski (1975) means that

$$
\int_{t}^{\infty} \bar{F}(u) d u \leq \mu e^{-\frac{t}{\mu}}, \quad \text { for all } t \geq 0
$$

This implies that

$$
\int_{0}^{\infty} \int_{t}^{\infty} \bar{F}(u) d u d t \leq \mu^{2}
$$

Which is equivalent to

$$
\int_{0}^{\infty} \mu(t) \bar{F}(t) d t \leq \mu^{2}
$$

Hence $m_{g}(F) \geq 0$, i.e., $\mathrm{F}$ has the PGM property.

The next result shows that any NBUMRL distribution has the PGM property.

Theorem 4.5. Let $F$ be NBUMRL distribution, then the coefficient of variation satisfies $C_{F}^{2} \leq 1$.

Proof. From Theorem 3.2 (ii), $F$ is NBUMRL iff

$$
\int_{t}^{\infty} \bar{F}(u) d u \leq \mu \bar{F}(t) \text {. Also } \mu(t) \bar{F}(t)=\int_{t}^{\infty} \bar{F}(u) d u .
$$

Therefore

$$
\begin{aligned}
E T^{2} & =2 \int_{0}^{\infty}\left(\int_{t}^{\infty} \bar{F}(u) d u\right) d t \\
& \leq 2 \int_{0}^{\infty} \mu \bar{F}(t) d t \leq 2 \mu^{2} .
\end{aligned}
$$

This proves the theorem.

Next, we show that the HNBUE property is inherited through the variability ordering.

Theorem 4.6. Let $F$ and $G$ be two life distributions with equal means. If $F<_{v} G$ and $G$ is $H N B U E$, then so is $F$.

Proof. Let the common mean be $\mu$. Note that

$$
\begin{array}{rlrl}
\int_{t}^{\infty} \bar{F}(u) d u & \leq \int_{t}^{\infty} \bar{G}(u) d u, \quad & \text { since } F<_{v} G \\
& \leq \mu e^{-\frac{1}{\mu}}, \quad \text { since } \mathrm{G} \text { is HNBUE. }
\end{array}
$$

Therefore $F$ is HNBUE. 
5. Virtual rate orderings.

Let $F$ and $G$ be two absolutely continuous life distributions with respective hazard rates $r_{F}(t)$ and $r_{G}(t)$. we shall assume that for any life distrbution $F, r_{F}(t)<\infty$ for all $t \geq 0$. Next we give the following:

Definition 5.1 The virtual rate $R(t)$ for any life distribution $F$ is given by

$$
R_{F}(t)=r_{F}(t)-r(0), \quad \text { for all } t \geq 0
$$

Now, we can give the following orderings.

Definition 5.2. Let $F$ and $G$ be two distributions as defined above. Then

(i) $F$ is said to be less virtual rate increasing ( $r i$ ) ordered than $G$, denoted by $F<_{\text {ri }} G$, if

$$
\left[R_{F}(t)-R_{G}(t)\right] \nearrow \text { in } t, \quad \text { for all } t \geq 0
$$

(ii) $F$ is 'ess new better than used virtual rate (nbur) ordered than $G$, denoted by $F<_{\text {nbur }} G$, if

$$
R_{F}(t) \geq R_{G}(t), \quad \text { for all } t \geq 0 .
$$

(iii) $\mathrm{F}$ is less averaged virtual rate increasing (ari) than $G$, denoted $F<_{\text {ari }} G$, if

$$
t^{-1} \int_{0}^{t}\left[R_{F}(u)-R_{G}(u) d u \nearrow \text { in } t \text { for all } t>0\right.
$$

(iv) $F$ is less new better than averaged used virtual rate (nbar) than $G$, denoted by $F<_{\text {nbar }} G$, if

$$
\int_{0}^{t} R_{F}(u) d u \geq \int_{0}^{t} R_{G}(u) d u . \quad \text { for all } t \geq 0 .
$$

Now we give without proof the following characterizations.

Theorem 5.3. Let $G(t)=1-\exp (-\lambda t)$ be an exponential distribution and let $F$ be any absolutely continuous distribution. Then

(i) $F<_{r i} G$ iff $F$ has the increasing failure rate (IFR) property.

(ii) $F<_{\text {nbur }} G$ iff $F$ has the new better than used in failure rate (NBUFR) property.

(iii) $F<_{a r i} G$ iff $F$ has the incerasing failure rate in average (IFRA) property.

(iv) $F<_{\text {nbar }} G$ iff $F$ has new better than averaged failure rate (NBAFR) property.

The IFR and IFRA classes of life distributions and their properties are found in Bryson and Siddiqui (1969). The NBUFR class is studied in Abouammoh and Ahmed (1988) and the NBAFR class is introduced in Loh (1984a,b).

The following implications between virtual hazard rate orderings can be verified in parallel to that of Theorem 3.3 of virtual age. 
Theorem 5.4. Let $F$ and $G$ be absolutely continuous life distybutions. Then

(i) $F<_{r_{i}} G \Longrightarrow F<_{\text {nbur }} G$,

(ii) $F<_{r i} G \Longrightarrow F<_{a r i} G \Longrightarrow F<_{n b a r} G$.

(iii) $F<_{\text {nbur }} G \Longrightarrow F<_{n b a r} G$

Using the definitions of virtual hazard ordering we obtain the following:

Theorem 5.5. Let $f$ and $g$ denote the densities of the life distributions $F$ and $G$, respectively. Then

(i) $F<_{\text {nbur }} G$ and $f(0) \geq g(0)$ or $g(0)=0$ implies that $F<_{h} G$.

(ii) $F<_{r i} G$ and $G=1-\exp (-\lambda t), \lambda>0, t \geq 0$ implies that $F$ is IFR.

(iii) $F<_{\text {ari }} G$ and $G$ as in par (ii) implies that $F$ is IFRA.

Note that the survival function $\bar{F}(t)$ can be expressed as

$$
\bar{F}(t)=\exp \left(-\int_{0}^{t} r(u) d u\right), \quad \text { for } \quad t \geq 0
$$

Then we can derive the condition for the equivalence of $F \leq_{s t} G$ and $F \leq_{n b a r} G$, where $F \leq_{s t} G$ is known in the literature by $F$ is less stochastically ordered than $G$.

Theorem 5.6. If $r_{F}(0)=r_{G}(0)<\infty$, then $F \leq_{n b a r} G$ iff $F \leq_{s t} G$.

Proof. The proof is straightforward and therefore omitted.

Let $F$ and $G$ be two absolutely continuous life distributions with respective failure rate functions $r_{F}(t)$ and $r_{G}(t)$. Gupta and Kirmani (1987) have shown that $r_{F} \leq r_{G}$ if and only if $\frac{\bar{F}}{G}$ is nondecreasing. In the following theorem we characterize the virtual rate increasing ordering via the log concavity of the ratio of the underlying survivals.

Theorem 5.7. $F<_{r i} G$ if and only if $\frac{\overline{\bar{F}}}{\bar{G}}$ is log concave.

Proof. $\bar{F} / \bar{G}$ is $\log$ concave

$\Longleftrightarrow w(t)=-\log \frac{\bar{F}(t)}{\bar{G}(t)}$ is convex

$\Longleftrightarrow \frac{d w(t)}{d t}=r_{F}(t)-r_{G}(t)$ is nondecreasing in $t, \forall t \geq 0$

$\Longleftrightarrow R_{F}(t)-R_{G}(t)$ is nondecreasing in $\mathrm{t}$ for all $t \geq 0$

$\Longleftrightarrow F<_{r i} G$.

6. Some miscellaneous results.

The following theorem interrelates the length biased random variable $T_{b}$, see Definition 2.4 , to the parent variable. 
Theorem 6.1. Let $T$ be a nonnegative random variable and $T_{b}$ be the corresponding length biased variable. Then

(i) $T_{b}<_{h} T$,

(ii) $T_{b}<_{m r 1} T$,

(iii) if $r(0)<\infty$, then $T_{b}$ is NBUFR.

(iv) if $T$ is NBUMRL, then $T_{b}$ is NBUMRL.

(v) if $T$ is IFR, then $T_{b}$ is IFR.

Proof. (i) Using relation (2.8), one can see that

$$
r_{b}(t)=\alpha(t) r(t)
$$

where $\alpha(t)=\left[\frac{t}{t+\mu(t)}\right] \leq 1, \forall t \geq 0$. Hence $r_{b}(t) \leq r(t)$.

(ii) Since $"<_{h} "$ implies " $<_{m r l}$ " the required result follows.

(iii) Since $r(0)<\infty$, then relation (2.8) implies that

$$
r_{b}(0)=0
$$

which means that $r_{b}(t) \leq r_{b}(0)$.

The proofs of parts (iv) and (v) are straightforward and hence omitted.

Next we see how the normalized mean residual life ordering inherits the NBUMRL property.

Theorem 6.2. Let $F<_{n m r 1} G$ and $G$ is NBUMRL, then $F$ is NBUMRL.

Proof. Since $F<_{n m r 1} G$, this means that

$$
\mu_{G} \mu_{F}(t) \leq \mu_{F} \mu_{G}(t)
$$

The NBUMRL property for the distribution $G$ means that $\frac{\mu_{G}(t)}{\mu_{G}} \leq 1$ and with (6.1) implies that $\mu_{F}(t) \leq \mu_{F}$.

Gupta and Kirmani (1987) proved, in similar context, that if $F<_{m r l} G$ and (6.1) holds, then $\bar{F} \leq \bar{G}$.

Kitchen and Proschan (1981) have shown that the fstatement "if the convolution of $\mathrm{n}$ IFRA units is exponential then $n-1$ units must have degenerate distributions at zero and the remaining unit is exponential" can be proved by showing that the class of nonnegative random variables with $C_{F} \leq 1$ is closed under sums where $C_{F}$ is the coefficient of variation for the underlying distribution. This statement was considered by Block and Savits (1979) for the IFRA distributions and by Shaked (1983) for the NBU distributions.

Following Kitchen and Proschan (1981) we can prove the following result. 
Theorem 6.3. If the convolution of $n$ NBUMRL units is exponential, then $n-1$ of the components must have degenerate distributions at zero and the remaining unit is exponential.

Proof. Let $X$ and $Y$ be nonnegative random variables (possibly dependent) with $E X \leq E Y<\infty, C_{F(x)} \leq 1, C_{F(y)} \leq 1$ and $C_{F(x+y)}=1$. Then, see Kitchen and Proschan (1981, Lemma 1), $x=\alpha y$ a.s., where

$$
\alpha= \begin{cases}E X / E Y, & \text { if } E Y>0 \\ 0 & \text { if } E Y=0\end{cases}
$$

The class of nonnegative random variables $C_{F} \leq 1$ is closed under sums. Let $X_{1}, \ldots, X_{n}$ are independent NBUMRL random variables. From Theorem 4.5, $C_{F\left(x_{i}\right)} \leq 1$, for all $i=1, \ldots, n$. Without loss of generality one can take $E X_{1} \leq \ldots \leq E X_{n}$. Let $S_{n}=X_{1}+\ldots+X_{n}$ be exponentially distributed. Note that $S_{n}-X_{n}$ and $X_{n}$ are linearly dependent and stochastically independent radom variables. Thus at least one of these two variables is degenerate at 0 . This yields that $X_{i} \equiv 0, i=1, \ldots, n-1$. Hence the remaining random variable $X_{n}$ must be exponential.

The following result characterizes the exponential distribution throuhg the nburordering.

Theorem 6.4. Let $\bar{F}^{*}(x)=\mu^{-1} \int_{x}^{\infty} \bar{F}(u) d u$ and let $F \geq_{n b u v} \operatorname{Exp}(\mu)$, where $\mu=\int_{0}^{\infty} \bar{F}(x) d x$. Then $F$ and $F^{*}$ have the same mean iff $F$ is exponential.

Proof. If $r^{*}($.$) is the hazard rate of F^{*}(\cdot)$ and $r^{*}(\cdot)<\infty$, then since

$$
\bar{F}^{*}(x)=\mu^{-1} \int_{x}^{\infty} \bar{F}(u) d u
$$

it follows that $\bar{F}^{*}(x)$ has density function $f^{*}$ given by

$$
f^{*}(x)=\frac{\bar{F}(x)}{\mu}, x \geq 0 .
$$

Therefore the hazard rate $r^{*}($.$) is expressed as$

$$
r^{*}(x)=\frac{f^{*}(x)}{\bar{F}^{*}(x)}=\frac{\bar{F}(x)}{\int_{x}^{\infty} \overline{\bar{F}}(u) d u}
$$

It follows that

$$
r^{*}(0)=\frac{\bar{F}(x)}{\int_{0}^{\infty} \bar{F}(u) d u}=\mu^{-1} \quad \text { and }
$$




$$
\begin{aligned}
r^{*}(0) \int_{0}^{\infty} \frac{F(u) d u}{r^{*}(u)} & =\mu^{-1} \int_{0}^{\infty} F(u)\left[\int_{u}^{\infty} \bar{F}(t) d t\right][\bar{F}(u)]^{-1} d u \\
& =\mu^{-1} \int_{0}^{\infty} \int_{u}^{\infty} \bar{F}(t) d t d u \\
& =\int_{0}^{\infty} \bar{F}^{*}(u) d u=\mu_{F^{*}} .
\end{aligned}
$$

Suppose now that $\mu_{F^{*}}=\mu_{F}$, then

$$
\int_{0}^{\infty} \frac{r^{*}(0)}{r^{*}(u)} \bar{F}(u) d u=\int_{0}^{\infty} \bar{F}(u) d u .
$$

This is equivalent to

$$
\int_{0}^{\infty} \frac{r^{*}(u)-r^{*}(0)}{r^{*}(u)} \bar{F}(u) d u=0 .
$$

Since $F$ is NBUMRL, then $F^{*}$ is NBUFR implying that $r^{*}(u)-r^{*}(0) \geq 0$. Thus

$$
\frac{r^{*}(u)-r^{*}(0)}{r^{*}(u)} \bar{F}(u)=0 \quad \text { a.s. }
$$

But the latter is equivalent to $r^{*}(u)=r^{*}(0)$. This means that $F^{*}$ is exeponential, or equivalently $F$ is exeponential.

On the other hand, if $F$ is exeponential, then $F s t=F^{*}$, and hence $\mu_{F}=\mu_{F}^{*}$.

Remark. The above theorem generalizes a result of Bhattacharjee (1982), since we use the weaker NBUE assumption.

Acknowledgement: The authors are grateful to the referee for some of his useful corrections and suggestions.

\section{References}

[1] Abouammoh, A. M. On the criteria of the mean remaining life. Prob. Statist. Lett., 6, (1988) 205-211.

[2] Abouammoh, A. M. and Ahmed, A. N., The new better than used failure rate class of life distributions. Adv. Appl. Prob., 19, (1988) 237-240.

[3] Bagai, I. and Kochar, S. C., On tail ordering and comparison of failure rates. Comm. Statist. Theory Meth., A-15, (1966) 1377-1388.

[4] Barlow, R. E. and Proschan, F., Statiatical Theory of Reliability and life Testing. To Begin With, Silver Spring MD (1981). 
[5] Bhattacharjee, M. C., The class of mean residual lives and some consequences. SIAM J. Discrete and Algebraic Meth., 5, (1982) 56-65.

[6] Block, H. W. and Savits, T. H., Systems with exeponential life and IFRA components lives. Ann. Statist., 7, (1979) $911-916$.

[7] Bryson, M. C., Discribing and testing for heavy tailed distribution. Proc. IMS/ASA joint meeting, (1974) 118-121.

[8] Bryson, M. C. and Siddiqui, M. M., Some criteria for aging. J. Amer. Statist. Assoc., 64, (1969) 1472-1483.

[9] Gupta, R. C. and Kirmani, S. N. U. A., On order relations between reliability measures. Comm. Statist. Stoch. Models, 13, (1987) 149-156.

[10] Gupta, R. C. and Keating, J. P., Relations for reliability measures under length biased sampling. Scand. J. Statist., 13 (1986) 49-56.

[11] Kitchen, J. and Proschan, F., Generalization of Block- Savits Convolution result. Ann. Statist., 9, 437 (1981).

[12] Kochar, S. C. and Wiens, D. P., Partial orderings of life distributions with respect to their ageing properties. Naval Res. Log. Qart., to appear (1987).

[13] Loh, W-Y, Bounds on AREs for restricted classes of distributions defined via tail orderings. Ann. Statist., 12, (1984a) 685-701.

[14] Loh, W-Y, A new generalization of the cless of NBU distributions. IEEE Trans. Reliability, R-33, (1984b), 419-422.

[15] Muth, E.J., Memory as property of probability distributions. IEEE Trans. Reliability, R-29, (1980) $160-164$.

[16] Rolski, T., Mean residual life. Bull. Int. Statist. Inst., 46, (1975) 266-270.

[17] Ross, S. M. and Schechner, Z., Some reliability applications of variability ordering. Oper. Res., 32, (1984) 679-687.

[18] Shaked, M., Exponential life functions with NBU componens. Ann. Prob., 11, (1983) $752-759$.

Department of Statistics, King Saud University, P.O. Box 2455, Riyadh 11431, Saudi Arabia. 